\title{
PARAMETERIZATION OF EVOLUTION OF BIPHASE DURING NONLINEAR TRANSFORMATION OF WAVES IN COASTAL ZONE
}

\author{
Yana Saprykina $^{1}$, Sergey Kuznetsov ${ }^{1}$ Margarita Shtremel $^{1}$
}

\begin{abstract}
Based on experimental data, the problem of parametrization of spatial variation of the phase shift (biphase) between the first and second nonlinear harmonics of wave motion during wave transformation over sloping bottom in the coastal zone is discussed. It is revealed that the biphase values vary in the range $[-\pi / 2, \pi / 2]$. Biphase variations rigorously follow fluctuations in amplitudes of the first and second harmonics and the periodicity of energy exchange between them. The empirical relation applied in modern practice to calculate the biphase, which depends on the Ursell number, is incorrect for calculating the biphase for wave evolution in the coastal zone, because it does not take into account periodic energy exchange between the nonlinear harmonics. The new approximations of the biphase values for typical scenarios of wave transformations are suggested. It was demonstrated that the biphase of breaking waves defines breaking index and breaking type.
\end{abstract}

Keywords: nonlinear wave transformation, nonlinear harmonics, biphase, wave breaking, coastal zone

\section{INTRODUCTION}

The evolution of waves in a coastal zone is weakly nonlinear dispersive process. It is characterized by growth of the highest multiple nonlinear harmonics of the main wave movement. Nonlinear harmonics arise as result of near resonant triad interactions which characteristic feature is the periodical exchange of energy between the first and highest harmonics. As a result of this process both their amplitudes and shifts of phases between them periodically change also. Shift of phases between the first and second nonlinear harmonics is described by the biphase. For example in simple model of free surface elevations with two nonlinear harmonics:

$$
\eta(x, t)=a_{1}(x) \cos \left(k_{1} x-\omega_{1} t+\varphi_{1}\right)+a_{2}(x) \cos \left(k_{2} x-\omega_{2} t+\varphi_{2}\right)
$$

where $\omega_{2}=2 \omega_{1}, k_{2}=2 k_{1}+\delta ; \mathrm{t}$ is time; $\omega$ is the angular frequency; $\mathrm{k}$ is the wavenumber, $\mathrm{a}_{1,2}$ is the amplitude; $\varphi_{1,2}$ is the phase; subscripts 1 and 2 correspond to the first and second harmonics; $\delta$ is detuning by the wavenumbers, determined by the dispersion relation and corresponding to nearresonance triad interactions occurring with spatial period $L_{b}$ (Madsen, Sorensen, 1993):

$$
L_{b}=2 \pi / \delta
$$

In this model biphase is $\beta=2 \varphi_{1}-\varphi_{2}$, (Elgar, Guza 1986).

For irregular waves, the biphase can be calculated by the following formula (Kim, Powers, 1976):

$$
\beta\left(\omega_{1}, \omega_{2}\right)=\arctan \left[\frac{\operatorname{Im}\left\{B\left(\omega_{1}, \omega_{2}\right)\right\}}{\operatorname{Re}\left\{B\left(\omega_{1}, \omega_{2}\right)\right\}}\right]
$$

where $B\left(\omega_{1}, \omega_{2}\right)=E\left[A_{\omega_{1}} A_{\omega_{2}} A_{\omega_{1}+\omega_{2}}^{*}\right]$ is the bispectrum, $\omega$ is the angular frequency, $A$ represents the complex Fourier amplitudes of the free surface elevations, and $E$ is the averaging operator. The frequencies of the first and second harmonics are determined by the positions of local maxima of the wave spectrum.

The biphase is characterized by a change in wave asymmetry, which influences on a direction of sediment transport in the coast zone (Bailard, Inman, 1981; Doering, Bowen, 1995). In Stokes waves, the biphase is equal to zero, since the positions of wave crests generated by the first and second nonlinear harmonics coincide (Hasselman et al., 1963).

Field studies of biphase variation in the coastal zone performed in (Elgar, Guza, 1985) revealed that it varies weakly around zero at a depth of around $9 \mathrm{~m}$ and is close to $-\pi / 2$ near the coast. Such variation in the biphase corresponded to observed changes in wave symmetry. In deep water, waves were almost symmetric in shape, but near the shore, they had a "saw-tooth" shape. Numerical simulation in (Elgar, Guza, 2986) showed that the initial phases of the nonlinear harmonics for quite

\footnotetext{
${ }^{1}$ V.P.Zenkovich Coast and Shelf Laboratory, P.P.Shirshov Institute of Oceanology, Russian Academy of Science, Nakhimovskii pr., 36, Moscow, 117995, Russia
} 
steep bottom slope of the coastal zone do not substantially influence the general trends of biphase variation: it is close to zero in relatively deep water and tends to $-\pi / 2$ near the coast in shallow water. The data of field experiments also showed that the evolution of the biphase is influenced by relative water depth and relative wave height (Doering, Bowen, 1986, 1995), but no clear dependence of the biphase values on these parameters has been obtained.

The values of a biphase are used at modeling of waves on spectral stochastic models and in SWAN model. For modeling a parametrization of change of a biphase on the Ursell number is used (Doering, Bowen, 1995; Eldeberky, Battjes , 1995):

$$
\beta=-\pi / 2+\pi / 2 \cdot \tanh (m / U r)
$$

$U r=(3 / 4) \cdot a k /(k h)^{3}-$ Ursell number, $\mathrm{m}=0.63$ or 0.2 - constant, $a$ - wave amplitude, $k$ - wave number, $h$ - water depth.

Eq.4 with $m=0.63$ is based on the results of analyzing the data of four field measurements of wave transformation at from four up to seven points of the coastal zone above bottom profiles with different mean inclinations, including a profile with underwater bar (Doering, Bowen, 1995). Eq.4 with $m=0.2$ is proposed as a result of analyzing data from a laboratory experiment on wave transformation with the JONSWAP spectrum with a spectral peak frequency of $0.2 \mathrm{~Hz}$ and a significant-wave height of $0.9 \mathrm{~m}$ above an even inclined bottom from a depth of $4.1 \mathrm{~m}$ (Eldeberky, Battjes , 1995). Note that the biphase values obtained in Eq.4 are always negative and are in the range $[0,-\pi / 2]$. For $\mathrm{Ur}<1$, they are close to zero, and for an increase in the Ursell number, they will tend to $-\pi / 2$.

In deriving empirical Eq.4, authors noted that not all the experimental data corresponded well to the given approximation (Doering, Bowen, 1995; Eldeberky, Battjes , 1995). The scatter of the biphase values for $U r<1$ in the field data ranged from $-\pi / 6$ to $\pi / 3$, and in the laboratory data, from $-\pi / 3$ to $\pi / 3$. For $U r \square 10$, deviations in the experimental data from Eq.4 are in the range $[-\pi / 18, \pi / 18]$ of the limiting value $-\pi / 2$. Nevertheless, despite such significant deviations, Eq. 4 with the constant $m=0.63$ is used in SWAN model to calculate the biphase values for $U r>1$, and for $U r<1$ the biphase is assumed equal to 0 (www.swan.tudelft.nl).

The existence of positive biphase values and deviation from Eq. 4 can be explained by the presence of periodic energy exchange between the first and second nonlinear harmonics during near-resonance triad interaction. The evolution of biphase and amplitudes during one period of nonlinear exchange of energy between harmonics was described in detail in (Kuznetsov, Saprykina, 2012). The second harmonic occurs with a phase shift of $-\pi / 2$ with respect to the first harmonic; for a certain time during which nonlinear processes predominate, the amplitude of the second harmonic increases, and the amplitude of the first harmonic decreases, the biphase remains close to this value. With an increase in amplitude of the second harmonic, the influence of dispersion processes intensifies, which leads to waves of the second harmonic lagging behind the first harmonic, as well as to a change in the biphase value from negative to positive. When the amplitude of the second harmonic is maximum, the biphase is equal to zero. Positive biphase values correspond to the back transfer of energy from the second harmonic to the first. Due to nonlinear process of back transfer of energy amplitude of second harmonics decreases practically to zero and due to dispersion process the biphase gradually becomes close to $+\pi / 2$. Thus, the periodic energy exchange between nonlinear harmonics can substantially influence the evolution of the biphase and variations in it occur in a wider range, from $-\pi / 2$ to $\pi / 2$, than are yielded by Eq.4.

The existence of periodical exchange at transformation of waves in a coastal zone was confirmed by data of field experiment four typical scenarios of wave transformation for the position of the maximum amplitude of the second harmonic was determined (Saprykina et al., 2009, 2013) :

1. the amplitude of the second harmonic increases significantly only near the coast;

2. the amplitude of the second harmonic reaches the maximum value within the studied area of the coastal zone and a starkly pronounced period of energy exchange between the first and second nonlinear harmonics is observed; Environmental fluctuations may have a negative effect on the number of species. This effect is due to physiological stress, which may even cause the extinction of some species;

3. the amplitude of the second harmonic varies weakly in the coastal zone; Environmental variability may have a positive effect on richness because it relaxes interspecific competition;

4. amplitudinally significant second harmonics already exist at the seaward boundary of the studied part of the coastal zone and half of the period of the energy exchange - backward energy transfer between the second and first harmonics - is observed. 
It was demonstrated also that realization of the scenarios depends on the relation between the local Iribarren number $-\xi=\alpha / \sqrt{H / L}$, where $\alpha$ is the mean bottom slope, $H$ is the local wave height, $L$ is the local wavelength, and on the wave steepness $(H / L)$ at the seaward boundary. The wave transformation scenarios with the pronounced periodic energy exchange between nonlinear harmonics ( 2 and 4$)$ will be, if $\xi<7 * H / L$. If $\xi>7 * H / L$, scenarios 1 and 3 will be realized.

The main purpose this work is to find out regularities of evolution of a biphase in the waves transforming over sloping bottom due to near resonant triad interactions and to investigate the possibility of parameterization of biphase variations.

\section{BIPHASE OF WAVES TRANSFORMING OVER AN INCLINED BOTTOM}

\section{Field experiment}

Wave transformation with the characteristic periodic energy exchange between the amplitudes of nonlinear harmonics was observed in the Shkorpilovtsy 2007 (Black sea, Bulgaria) field experiment for wave propagation over gentle sloping bottom profile with underwater bar (Fig. 1).

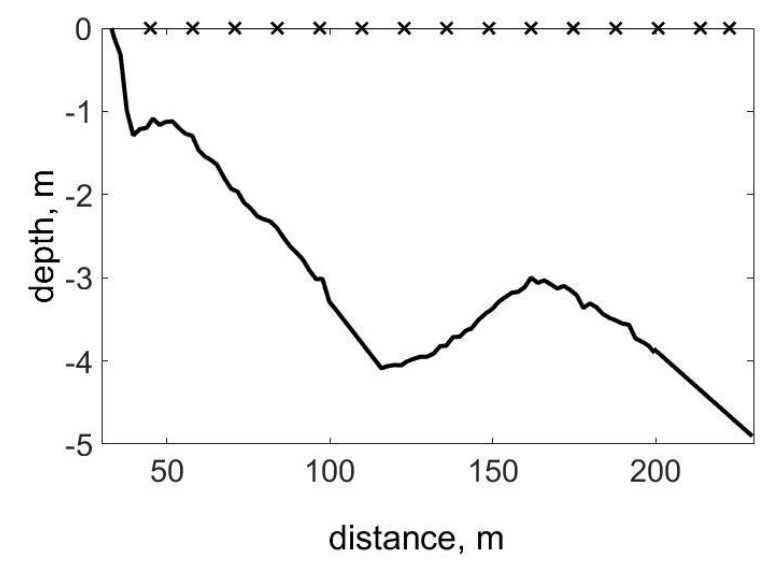

Figure 1. Bottom profile and positions of wave gauges (x), field experiment Shkorpilovtsy 2007.

During the experiment 7 resistance and 8 capacity wire type gauges were installed for synchronous registration of free surface elevation at sampling rate from 5 to $20 \mathrm{~Hz}$. The mean bottom slope was 0.022. Duration of wave records was from $20 \mathrm{~min}$ up to 1 hour and total 65 wave records were measured.

All analyzed regimes of waves on the seaward wave gauge correspond to condition

$$
0.095<h / L<0.3
$$

where $\mathrm{h}$ - water depth and $\mathrm{L}$ - wave length on the seaward wave gauge.

As the wave height, the significant wave height was calculated:

$$
H_{\text {sig }}=4 \sqrt{m_{0}} \text {, }
$$

where $m_{0}=\int_{0}^{\infty} S(\omega) d \omega ; S$ is the wave spectrum.

The period was determined as the period of the spectral maximum. In Table 1 are parameters of waves of four characteristic scenarios of wave transformations.

\begin{tabular}{|l|l|l|l|l|l|l|}
\hline \multicolumn{7}{|c|}{ Table 1. Wave parameters of field experiment } \\
\hline \begin{tabular}{l} 
No. of $\begin{array}{l}\text { Period, } \\
\text { measurement } \\
\text { series }\end{array}$ \\
\hline $7, \mathrm{~s}$
\end{tabular} & $\begin{array}{l}\mathrm{H}, \mathrm{m} \\
\mathrm{H} / \mathrm{L}\end{array}$ & $\begin{array}{l}\text { Iribarren } \\
\text { number, } \xi\end{array}$ & $\begin{array}{l}\text { Mean bottom } \\
\text { slope, } \mathrm{a}\end{array}$ & $\begin{array}{l}\text { Wave } \\
\text { transformation } \\
\text { scenario }\end{array}$ \\
\hline 28 & 5.9 & 0.55 & 0.014 & 0.194 & 0.022 & 1 \\
\hline 34 & 4.5 & 0.64 & 0.0212 & 0.156 & 0.022 & 3 \\
\hline 60 & 6.9 & 1.16 & 0.0252 & 0.144 & 0.022 & 2 \\
\hline
\end{tabular}


The biphase values for wave regimes of the field experiment are close to Eq.4 with the constant 0.63 only for $\mathrm{Ur}>1$ (scenarios 1,2 , and 4). The biphase values for $U r<1$ are not described by this approximation. The change in the biphase in scenario 3 does not entirely coincide with Eq.4. For $U r<$ 1, positive biphase values are observed, related to the periodic energy exchange and not described by Eq.4 (Fig. 2a).
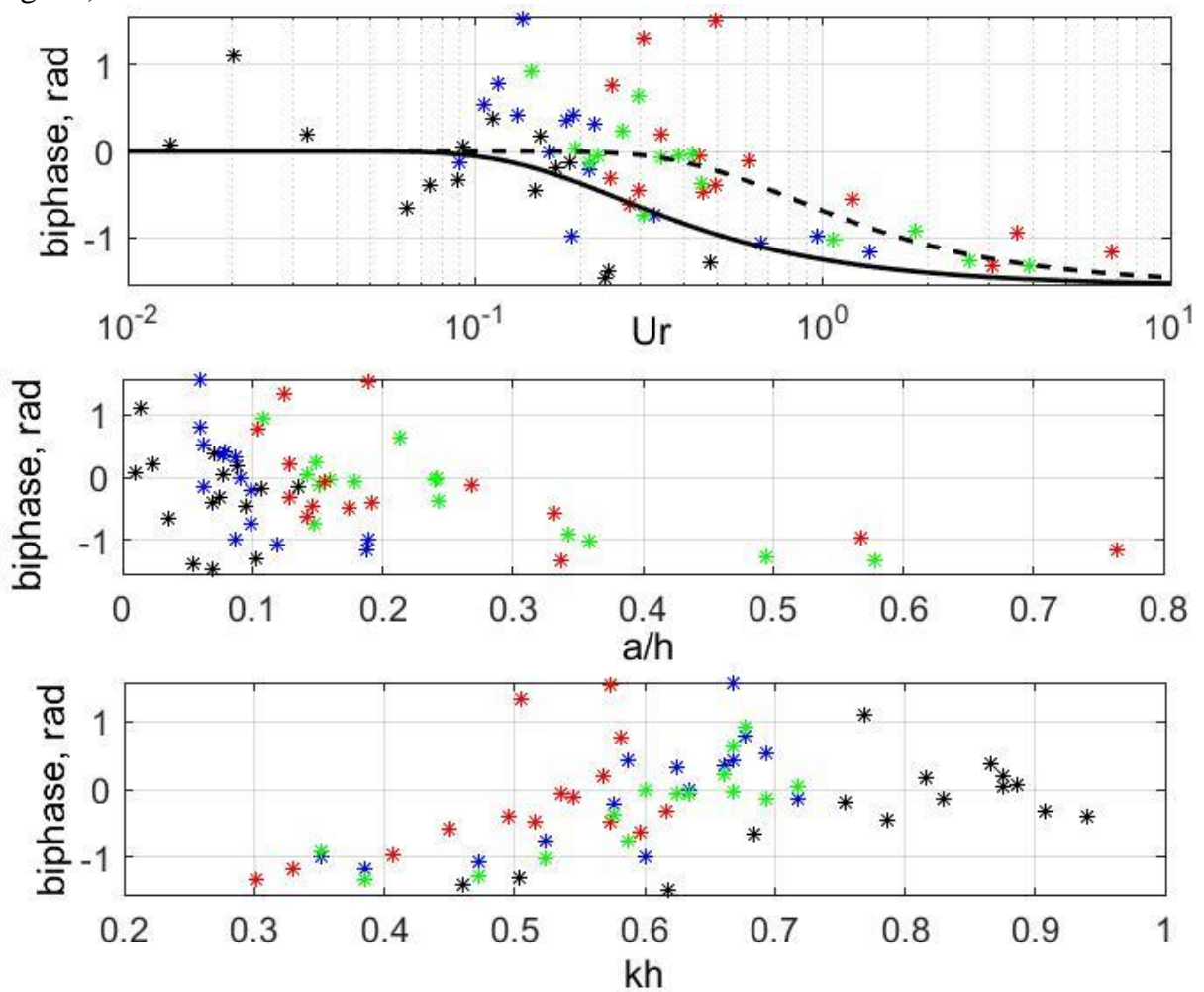

Figure 2. Dependences of biphase on Ursell number (a), kh (b) and ah (c) for different scenarios of wave transformations in field experiment Skorpilovtsy 2007: scenario 1 - blue, scenario 2 - red, scenario 3 black, scenario 4 - green. Solid line Eq. 4 with $m=0.2$, dotted line - with $m=0.63$.

Figures $2 \mathrm{~b}$ and $2 \mathrm{c}$ show the change in the biphase as a function of nonlinearity parameter $a / h$ and dispersion parameter $k h$. The wave amplitudes $(a)$ were estimated as half the height of significant waves. A clear dependence on the nonlinear or dispersion parameter is not observed. However, one can see that for all wave transformation scenarios, the biphase values vary in the range $[-\pi / 2, \pi / 2]$ for $a / h<$ 0.3 and they are negative for $a / h>0.3$. For $a / h>0.4$, when the relative wave height is close to the limiting height of breaking waves, the biphase values for scenarios 2 and 4 are close to $-\pi / 2$ (Fig. $2 b$ ). For $k h<0.5$, the biphase values are also negative for all wave transformation scenarios and tend to $-\pi / 2$ with a further decrease in $k h$. One can see that the negative biphase values for scenario 1 are observed starting from $k h<0.55$, and for scenarios 2-4, starting from a $k h$ less that $0.5,0.75$, and 0.65 , respectively. For $0.5<k h<0.8$, the biphase varies in the range $[-\pi / 2, \pi / 2]$. For $k h>0.8$, the range of biphase variations narrows to $[-\pi / 6, \pi / 6]$ (Fig. 2c).

\section{Laboratory Experiment}

To obtain experimental data with other initial wave parameters and bottom slopes necessary for a detailed study of the evolution of the biphase in the presence of periodic energy exchange between the first and second nonlinear harmonics for wave transformation above an inclined bottom, a laboratory experiment was performed in a hydraulic flume of Research center "Sea Coasts" (Sochi, Russia) in July 2013. The length of flume is $22 \mathrm{~m}$, the width $-0.6 \mathrm{~m}$, the depth $-1 \mathrm{~m}$. Physical modeling was carried out on the scale of 1:20 to natural processes. The wave chronograms were recorded synchronously at 14 points by digital capacity type wire gauges (Fig. 3). The evolution of pseudo monochromatic waves above two bottom slopes - 0.022 (gentle) and 0.043(steep) was investigated. Total during the experiment 65 wave regimes were tested. The similarity of model and field processes was evidenced by equality of the Froude numbers. 


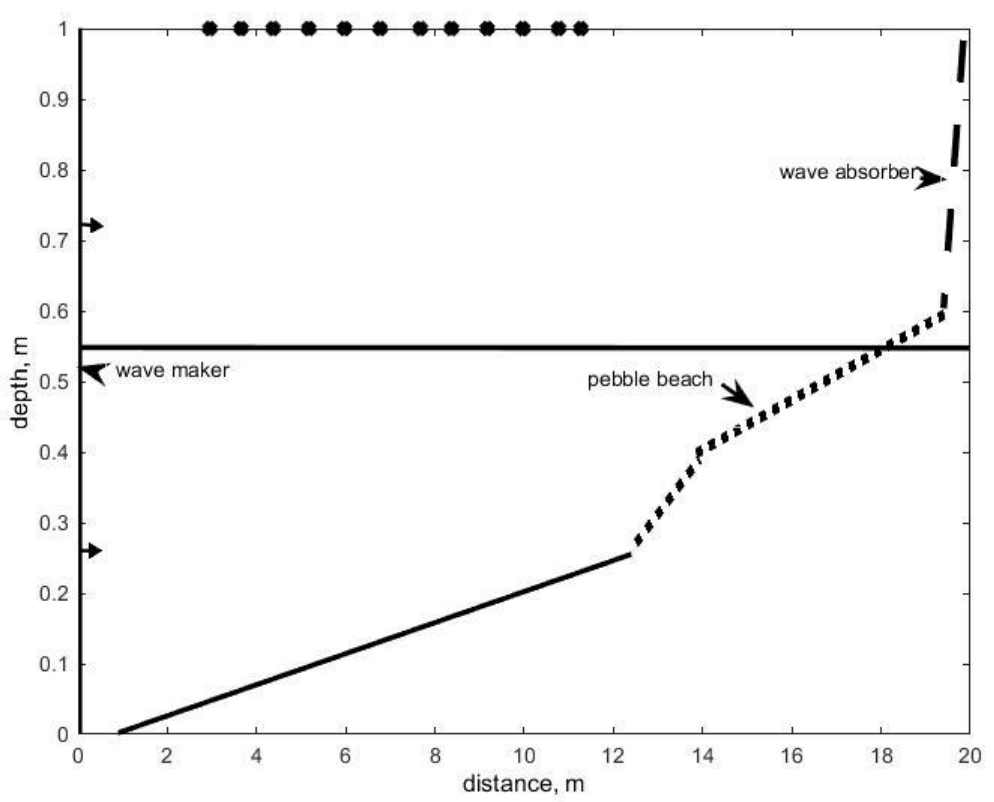

Figure 3. Bottom profile and positions of wave gauges (x), laboratory experiment, bottom slope 0.043 .

The initial water depth near the wave generator in the experiments was $0.55 \mathrm{~m}$ for a bottom inclination of 0.043 and $0.34 \mathrm{~m}$ for an inclination of 0.022 . In the experiment, the main focus was to obtain wave transformation scenarios with a starkly pronounced periodic energy exchange between the nonlinear harmonics (2 and 4) for a wider set of initial wave parameters than in the field experiment for an in-depth analysis of the change in the biphase. For all experimental regimes, the initial conditions determined at the point of the onset of wave transformation above an underwater slope, just like in the field data, corresponded to condition (4).

In Table 2 are the parameters of laboratory waves used for analysis and scenarios of its transformations defined by relations between wave steepness and Iribarren number mentioned above and confirmed by spectral analysis of evolution of amplitudes of first and second harmonics.

\begin{tabular}{|l|l|l|l|l|l|l|}
\hline \multicolumn{7}{|c|}{ Table 2. Wave parameters of lab experiment } \\
\hline $\begin{array}{l}\text { No. of } \\
\text { measurement } \\
\text { series }\end{array}$ & $\begin{array}{l}\text { Period } \\
T, \mathrm{~s}\end{array}$ & $\begin{array}{l}\text { Height } \\
\mathrm{H}, \mathrm{m}\end{array}$ & $\begin{array}{l}\text { Steepness } \\
\mathrm{H} / \mathrm{L}\end{array}$ & $\begin{array}{l}\text { Iribarren } \\
\text { number, } \xi\end{array}$ & $\begin{array}{l}\text { Bottom } \\
\text { slope, } \\
\mathrm{a}\end{array}$ & $\begin{array}{l}\text { Wave } \\
\text { transformation } \\
\text { scenario }\end{array}$ \\
\hline 6 & 2.56 & 0.09 & 0.0151 & 0.3494 & 0.043 & 1 \\
\hline 7 & 1.86 & 0.15 & 0.0347 & 0.2307 & 0.043 & 2 \\
\hline 8 & 1.46 & 0.276 & 0.0814 & 0.1507 & 0.043 & 2 \\
\hline 9 & 1.08 & 0.22 & 0.0877 & 0.1452 & 0.043 & 4 \\
\hline 10 & 0.93 & 0.153 & 0.0709 & 0.1615 & 0.043 & 4 \\
\hline 11 & 1.07 & 0.228 & 0.0918 & 0.1419 & 0.043 & 2 \\
\hline 12 & 1.37 & 0.189 & 0.0594 & 0.1764 & 0.043 & 4 \\
\hline 13 & 1.7 & 0.172 & 0.0436 & 0.2060 & 0.043 & 2 \\
\hline 15 & 2.56 & 0.119 & 0.0200 & 0.3039 & 0.043 & 1 \\
\hline 17 & 1.71 & 0.13 & 0.0327 & 0.2376 & 0.043 & 2 \\
\hline 18 & 1.365 & 0.11 & 0.0346 & 0.2312 & 0.043 & 2 \\
\hline 19 & 1.08 & 0.15 & 0.0598 & 0.1758 & 0.043 & 2 \\
\hline 20 & 0.99 & 0.07 & 0.0305 & 0.2464 & 0.043 & 3 \\
\hline 48 & 1.86 & 0.157 & 0.0462 & 0.1023 & 0.022 & 2 \\
\hline 49 & 1.86 & 0.13 & 0.0383 & 0.1124 & 0.022 & 2 \\
\hline 50 & 1.86 & 0.1 & 0.0295 & 0.1282 & 0.022 & 2 \\
\hline 51 & 1.86 & 0.12 & 0.0353 & 0.1170 & 0.022 & 2 \\
\hline 53 & 1.46 & 0.16 & 0.0600 & 0.0898 & 0.022 & 4 \\
\hline 54 & 1.46 & 0.13 & 0.0488 & 0.0996 & 0.022 & 2 \\
\hline 55 & 1.46 & 0.114 & 0.0428 & 0.1064 & 0.022 & 2 \\
\hline 56 & 1 & 0.12 & 0.0657 & 0.0858 & 0.022 & 4 \\
\hline 58 & 2.3 & 0.15 & 0.0357 & 0.1164 & 0.022 & 2 \\
\hline
\end{tabular}


Figure 4 shows the changes in the biphase for different wave transformation scenarios above a steep bottom $(0.043)$ as a function of the Ursell number and the dispersion $(k h)$ and nonlinear $(a / h)$ wave parameters.
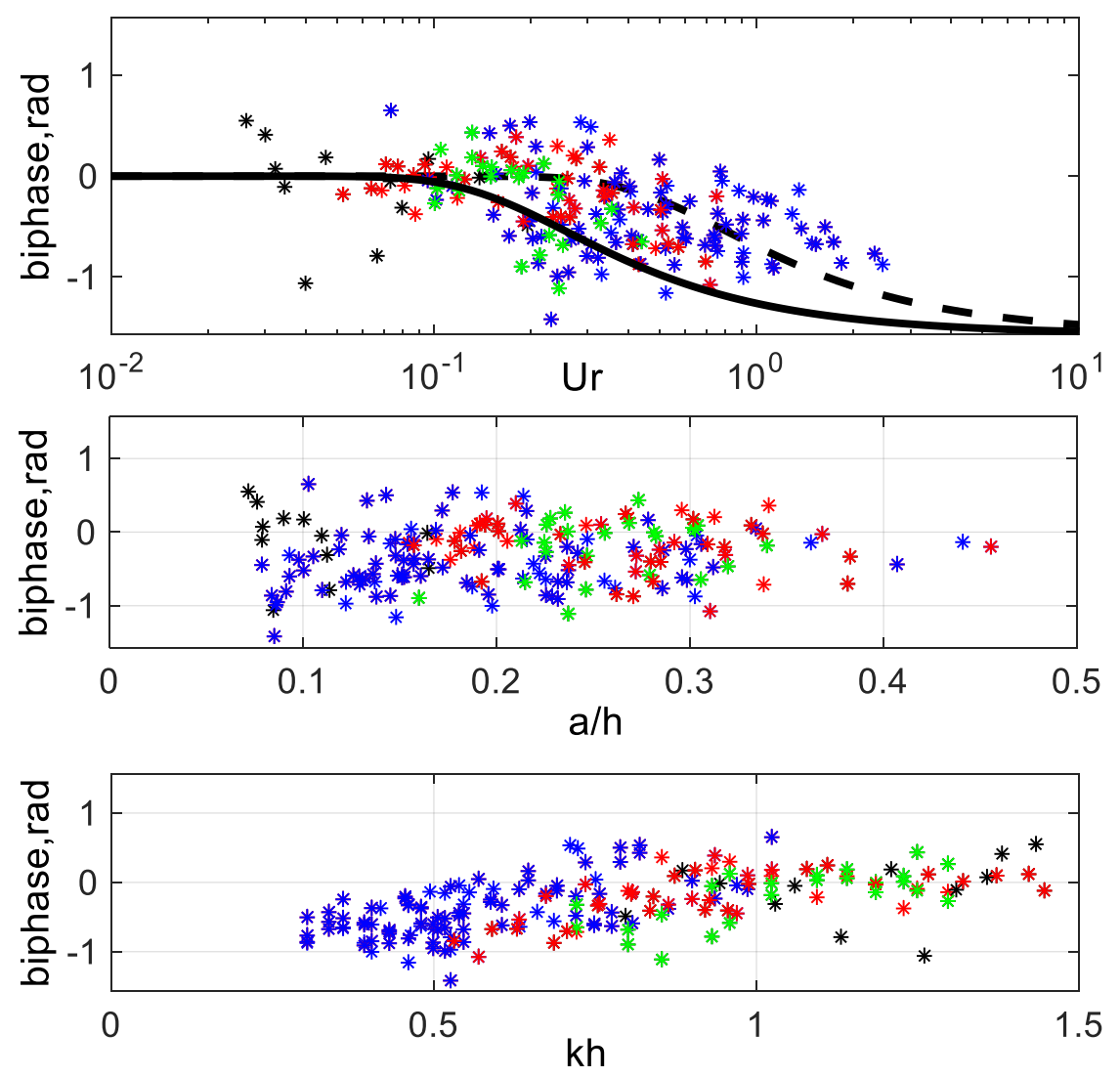

Figure 4. Dependences of biphase on Ursell number (a), kh (b) and ah (c) for different scenarios of wave transformations in laboratory experiment Sochi 2013: scenario 1 - blue, scenario 2 - red, scenario 3 - black, scenario 4 - green. Solid line Eq. 4 with $\mathrm{m}=0.2$, dotted line - with $\mathrm{m}=0.63$. Bottom slope 0.043 .

It is visible that the biphase values are poorly described by Eq.4 for all wave transformation scenarios. Both positive and negative biphase values are observed for $0.02<U r<0.9$. Only for scenario 1 for $U r>1$ did the biphase values qualitatively follow Eq.4 with $m=0.63$. Variation in the biphase for all scenarios hardly depends at all on nonlinear parameter $a / h$. One can also see that, just like in the field experiment data, for $a / h>0.37$, when the height of waves is close to their limit height of breaking waves, the biphase values are always negative.

For all the scenarios, a general trend of biphase variation depending on the dispersion parameter $k h$ is traced: for $k h<0.6$, the biphase is negative and lies in the range from $-\pi / 2$ to 0 . There is no starkly pronounced dependence on the type of wave transformation scenario, and one can also see that for scenarios 2 and 4, the biphase becomes negative, starting at approximately $k h<0.7$ and 0.9 , respectively.

For wave transformation above a gently sloping bottom (0.022), the evolution of the biphase occurs somewhat differently (Fig.5). Note that for the chosen initial parameters of the experimental waves (Table 2), their transformation scenarios 1 and 3 are not observed; more often than not, scenario 2 is realized, with one total period of energy exchange between the first and second nonlinear harmonics. Figure 5 shows the dependences of the biphase on the Ursell number and the dispersion $(k h)$ and nonlinear $(a / h)$ wave parameters for gentle slope $(0.022)$. The biphase values, just like in the case of a steep slope, are poorly described by Eq.4. The biphase values can be positive for $0.1<U r<2$. Just like in the case of wave transformation above a bottom with slope 0.043 , variation in the biphase hardly 
depends at all on nonlinear parameter $a / h$ except for waves close to the limit height of breaking wave, for which for $a / h>0.37$ the biphase will be negative (Fig. $5 b$ ).

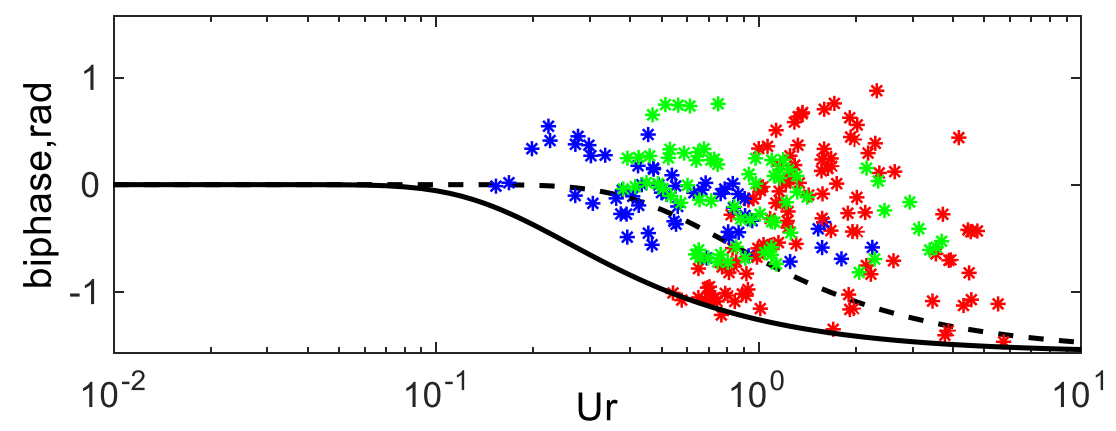

a

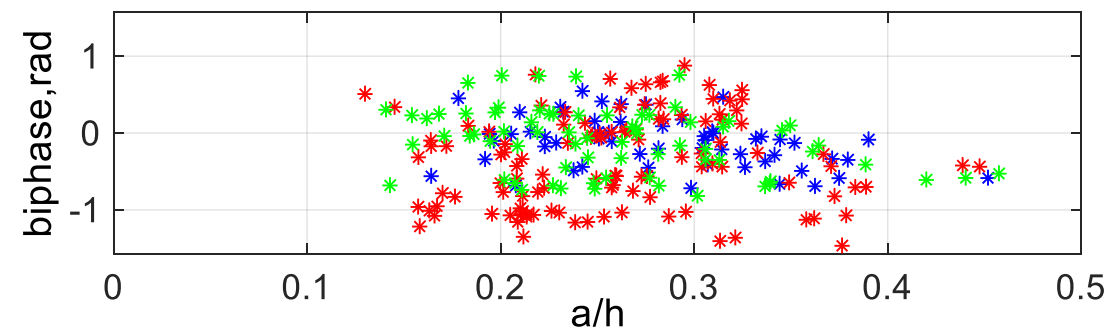

b

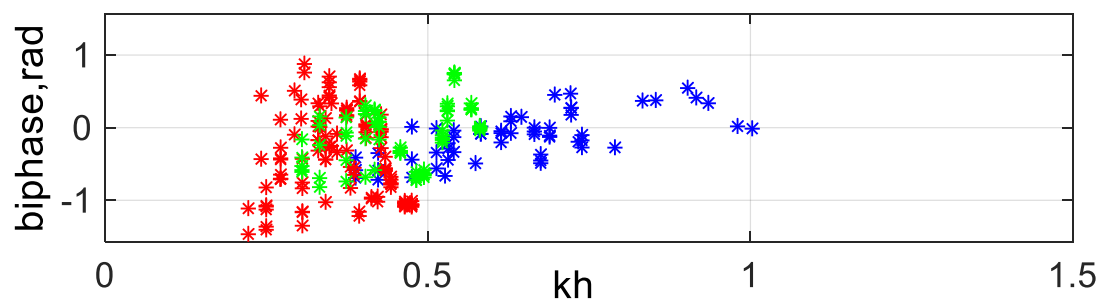

c

Figure 5. Dependences of biphase on Ursell number (a), kh (b) and ah (c) for different scenarios of wave transformations in laboratory experiment Sochi 2013: scenario 1 - blue, scenario 2 - red, scenario 3 - black, scenario 4 - green. Solid line Eq.4 with $\mathrm{m}=0.2$, dotted line - with $\mathrm{m}=0.63$. Bottom slope 0.022 .

A certain dependence of the biphase on the dispersion parameter is observed: for $k h<0.35$ the biphase is negative and lies in the range from $-\pi / 2$ до 0 . In the case of a gently sloping bottom with an inclination of 0.022 , these biphase values will be observed for a $k h$ less than for wave transformation above a steep bottom. For $0.6<k h<0.4$, the biphase, in addition to negative values, has substantial positive values, which is related to the periodicity of the energy exchange between the harmonics. For $k h$ values close to 1 , the biphase is close to zero (Fig. $5 \mathrm{c}$ ).

Thus, Eq.4 poorly describes changes in the biphase in the case of wave propagation above an inclined bottom for all of the wave transformation scenarios. To correctly parameterize the biphase, it is necessary to take into account the periodicity of energy exchange between the harmonics; i.e., the wave transformation scenario can play a significant role. For all bottom inclinations, the evolution of the biphase depends on $k h$ : we can say that for $k h<0.35$, the biphase is negative and ranges from $\pi / 2$ to 0 , and for $k h>1$, the mean biphase values are close to zero. Independently of bottom slope, the biphase for $a / h>0.37$ (waves of the limiting height of breaking waves) becomes negative. According to the laboratory experiment, no change in the biphase dependent on the nonlinear parameter for $a / h<0.37$ was revealed. The bottom inclination and wave transformation scenario affect the dependence of the change in the biphase on dispersion parameter $k h$.

Note that in all the studied experimental cases, the change in the biphase occurred in the range from $-\pi / 2$ to $\pi / 2$ (Figs. 4,5 ). An important consequence of such a change in the biphase is that the wave component of load dispersion in models based on the Bagnold concept (Bagnold, 1966), which estimate sediment discharge using wave velocity asymmetry (e.g., in Antsyferov et al., 2005), will always be directed toward the coast, because its value is proportional to the cosine of the biphase. 


\section{PARAMETERIZATION OF CHANGES IN THE BIPHASE TAKING INTO ACCOUNT PERIODIC ENERGY EXCHANGE BETWEEN THE HARMONICS}

Analysis of changes in the biphase in the experimental data has shown that its evolution strictly follows changes in the amplitudes of the harmonics, in accordance with the direction of energy exchange between them (for example, Fig.6). As was shown in the Introduction, the spatial period of energy exchange occurs at distance $L_{b}(2)$, which increases with decreasing depth, because the value of the detuning $\delta$ decreases.
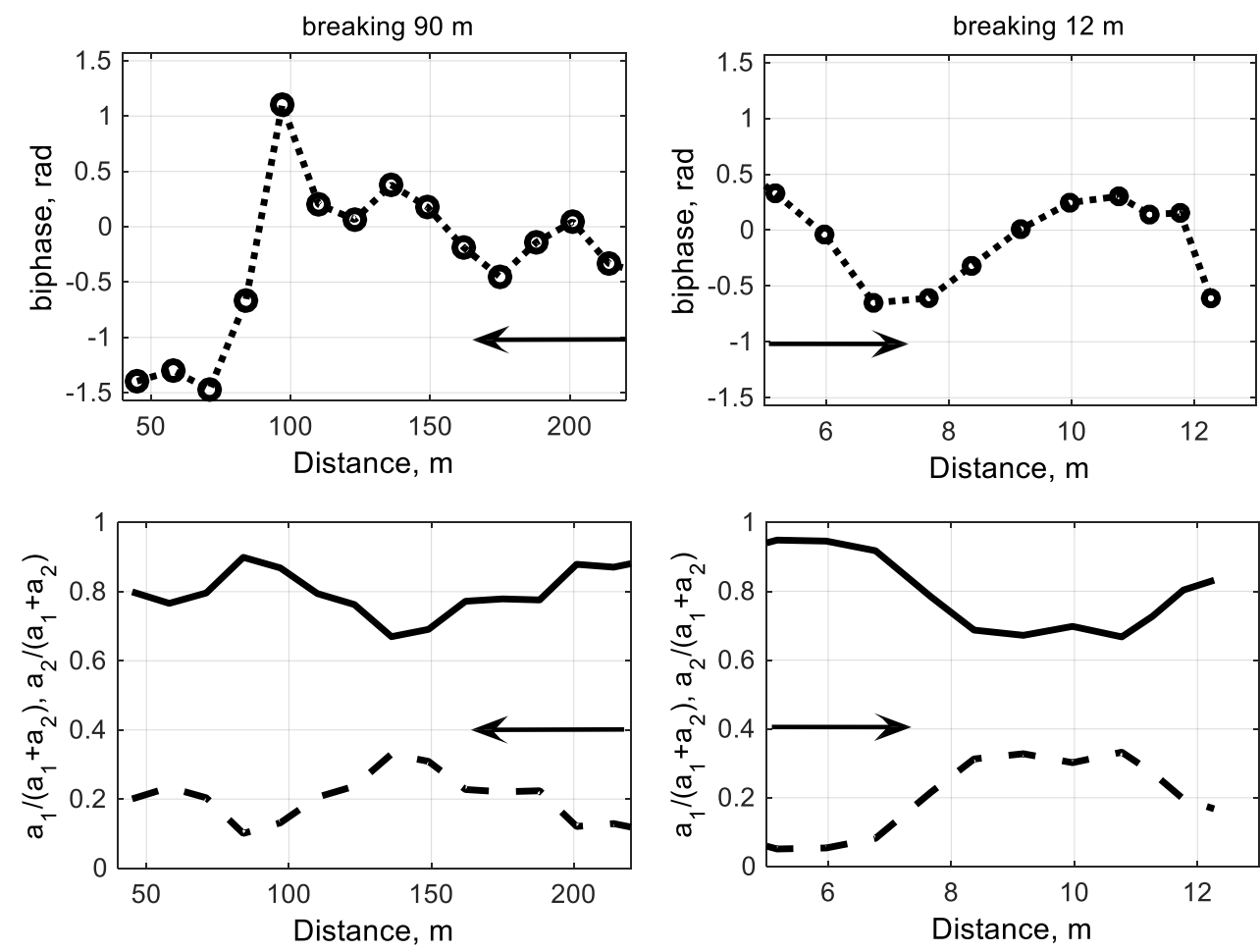

Figure 6. Example of typical changes of biphase and relative amplitudes of first (solid line) and second (dashed line) nonlinear harmonics for scenario 2 in field (left) and laboratory (right) experiments. The arrows show direction of waves propagation.

Let us consider the evolution of the biphase from a parameter that takes into account the possible number of periods of energy exchanges for wave propagating from some local water depth to a shore. As such a parameter - relative wave run, we will take the ratio of the distance to the coast $(h / \alpha)$ to the expected spatial length of the period of energy exchange between the first and second nonlinear harmonics $L_{b}$ :

$$
\Delta l=(h / \alpha) / L_{b},
$$

where $h$ is the local water depth, $\alpha$ is the local mean bottom slope, and $L_{b}$ is from Eq.2.

To determine the detuning of the wavenumbers $\delta=k_{2}-2 k_{1}$, the wavenumbers of the first $\left(k_{1}\right)$ and second nonlinear harmonics $\left(k_{2}\right)$ are calculated by the dispersion relation of linear wave theory.

Figure 7 shows the changes of the biphase in the experimental data from the proposed parameter $\Delta l$. One can see that for all analyzed cases, nearly the same change in the biphase occurs: weak fluctuations with respect to zero at distances to the coast larger than five exchange periods $L_{b}$, then a gradual broadening of the range of changes in the biphase to an interval of $[-\pi / 2, \pi / 2]$ at distances to the coast within one to three of the proposed exchange periods $L_{b}$, and a subsequent narrowing of this interval up to changes primarily in the negative domain in the interval $[-\pi / 2,0]$ at distances to the coast on the order of one $L_{b}$. The largest range of possible biphase values in the interval from $[-\pi / 2, \pi / 2]$ will be observed for $1<\Delta l<3$. For $\Delta l<0.5$, the biphase for all scenarios of the experimental data is strictly negative and tends to $\kappa-\pi / 2$, and an approximation of the upper limit of its changes exists:

$$
\beta=\pi / 2 * \Delta l-\pi / 2
$$




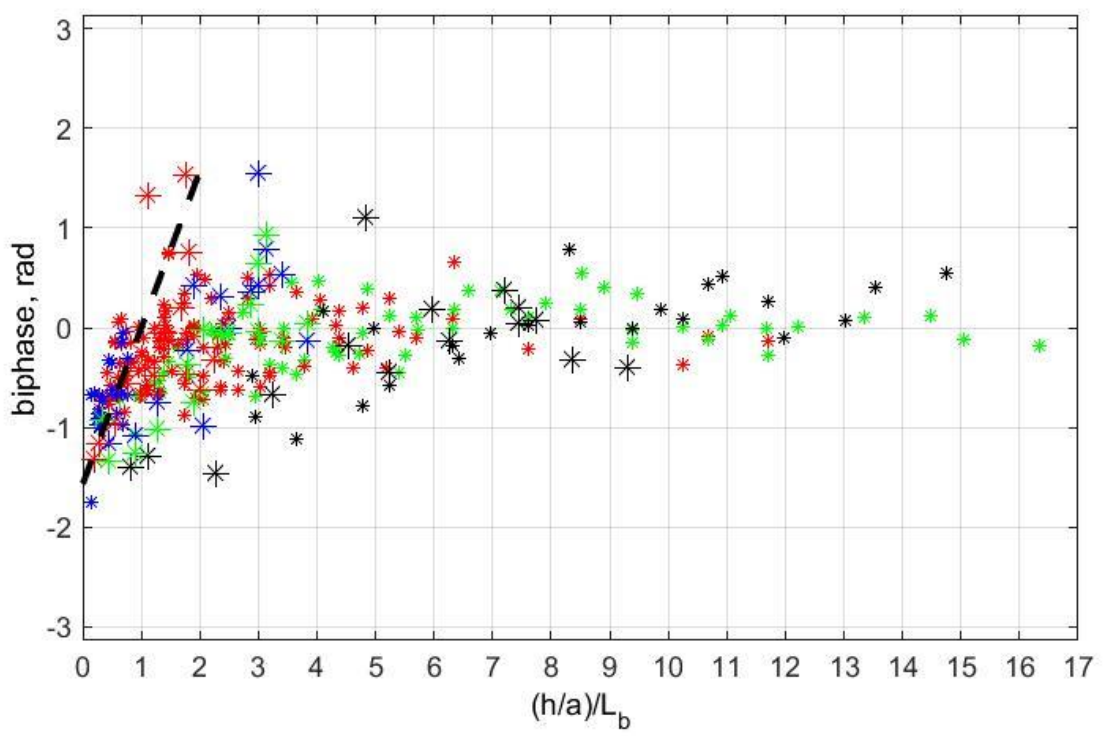

Figure 7. Dependence of the biphase on relative wave run: scenario 1 - blue, scenario 2 - red, scenario 3 black, scenario 4 - green. Large stars correspond to data of field experiment.
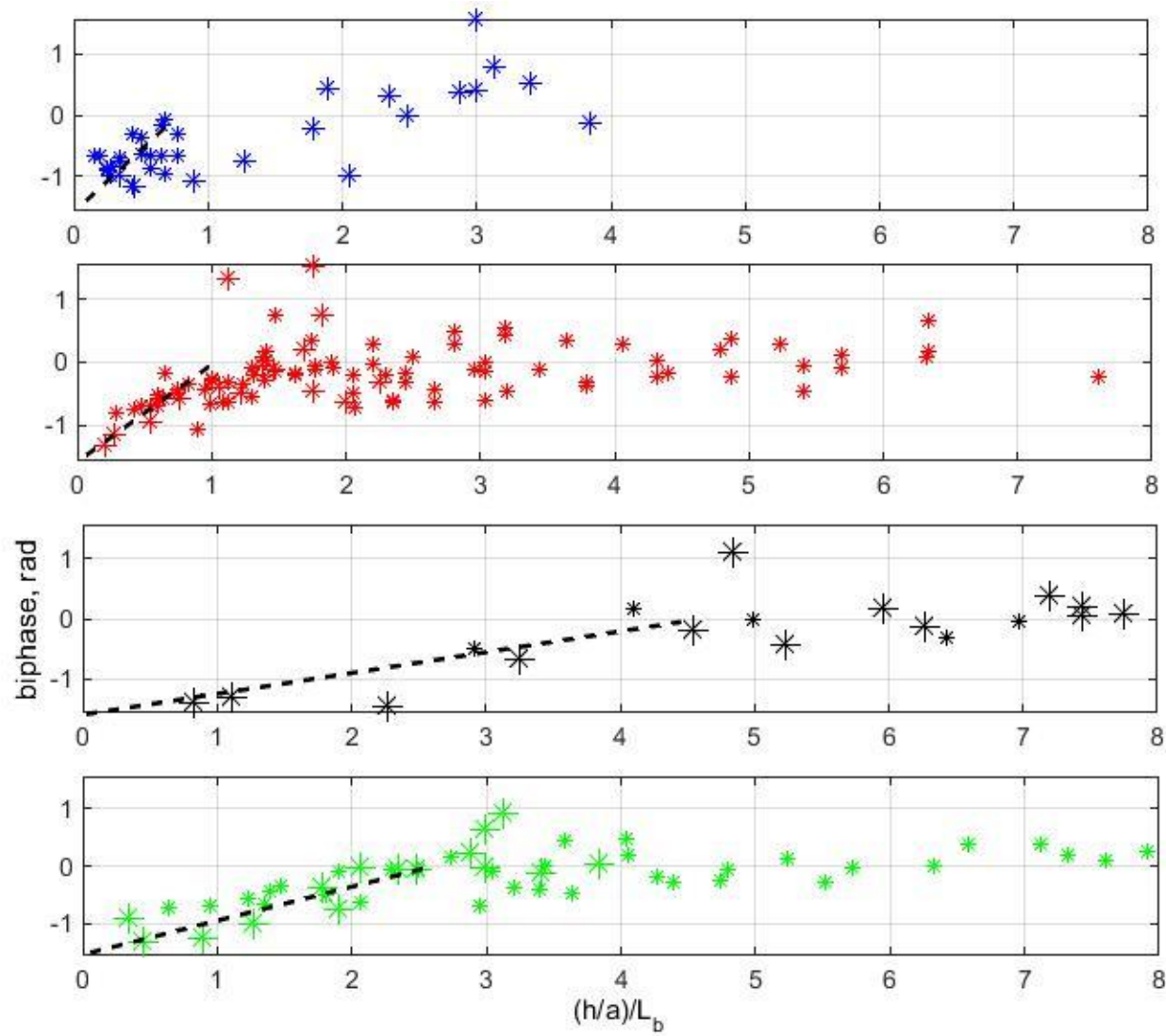

Figure 8. Dependences of the biphase on relative wave run for different scenarios of wave transformation: scenario 1 - blue, scenario 2 - red, scenario 3 - black, scenario 4 - green. Large stars correspond to data of field experiment. 
Thus, using parameter $\Delta l$, a nearly general approximation of the biphase values is possible for wave propagation above an inclined bottom: for $\Delta l>5$, the biphase is taken as equal to zero; for $\Delta l<1$, the biphase is approximately determined by the change in its upper limiting values by formula (10). For $1<$ $\Delta l<5$, a general formula for parameterizing changes in the biphase is impossible.

When analyzing available experimental lab and field data, we observed a dependence of changes in the biphase on parameter $\Delta l$ and on the wave transformation scenario (Fig. 8). For example, negative values of the biphase changes for scenario1 at $\Delta l<0.5$ can be approximated by a linear dependence Eq.8. But for scenario 2 negative biphases are at $\Delta l<1$, and it can be approximated as

$$
\beta=\pi^{*} \Delta l-\pi / 2
$$

For scenario 3, negative changes in the biphase begin for $\Delta l<4$ and they can be approximated as

$$
\beta=\pi / 9 * \Delta l-\pi / 2
$$

For scenario 4 , negative biphases are for $\Delta l<2.5$ and they can be approximated by the dependence

$$
\beta=\pi / 5 * \Delta l-\pi / 2
$$

However, the dependence of variations in the biphase on the transformation scenario and the proposed Eq.9-11 require further verification using field measurement data.

\section{BIPHASE OF BREAKING WAVES}

Wave breaking is a strongly nonlinear process and is characterized by a decrease in wave height. As was demonstrated in (Kuznetsov, Saprykina, 2004), during breaking, a strongly pronounced frequency selectivity of wave energy dissipation can be observed: breaking changes the wave spectrum, compensating the nonlinear increase in the higher harmonics, causing an intense decrease in the amplitudes of the second harmonics, which can also affect the change in the biphase. According to the most popular concept on wave evolution in the coastal zone, waves before breaking assume a saw-tooth shape and the biphase in them is close to $-\pi / 2$ ( for example, Elgar, Guza, 1985, 1986). But the waves breaking by different types, for example, in coastal zone plunging (Pl) and spilling (Sp) breaking types more often are realized. Plunging and spilling breaking waves have different symmetry against vertical axis: plunging breaking waves are asymmetrical and have a saw-tooth shape, but spilling breaking waves are practically symmetrical (Kuznetsov, et al., 2015). The symmetry of waves against vertical axis is defined by asymmetry coefficient

$$
\text { As }=\frac{\left\langle\eta_{\mathrm{H}}^{3}\right\rangle}{\sigma^{3}}
$$

\begin{tabular}{|c|c|c|c|c|}
\hline $\begin{array}{l}\text { No. of } \\
\text { measurement } \\
\text { series }\end{array}$ & $\begin{array}{l}\text { Type of } \\
\text { wave } \\
\text { breaking }\end{array}$ & $\begin{array}{l}\text { bottom } \\
\text { slope }\end{array}$ & $\begin{array}{l}\text { Period, } \\
T, \mathrm{~s}\end{array}$ & $\mathrm{v}=H_{s} / h_{b}$ \\
\hline 7 & Sp & 0.043 & 2.56 & 0.60 \\
\hline 8 & Sp & - & 1.46 & 0.76 \\
\hline 10 & Sp & - & 0.93 & 0.67 \\
\hline 11 & Sp & - & 1.00 & 0.68 \\
\hline 12 & $\mathrm{PI}$ & - & 1.36 & 0.90 \\
\hline 13 & $\mathrm{PI}$ & - & 1.70 & 0.81 \\
\hline 15 & Sp & - & 2.56 & 0.57 \\
\hline 17 & Sp & - & 1.70 & 0.59 \\
\hline 19 & Sp & - & 1.00 & 0.68 \\
\hline 47 & Sp & 0.022 & 2.56 & 0.64 \\
\hline 48 & Sp & - & 1.86 & 0.68 \\
\hline 49 & Sp & - & 1.86 & 0.63 \\
\hline 51 & Sp & - & 1.86 & 0.59 \\
\hline 52 & Sp & - & 1.86 & 0.51 \\
\hline 53 & Sp & - & 1.46 & 0.78 \\
\hline 54 & Sp & - & 1.46 & 0.70 \\
\hline 55 & Sp & - & 1.46 & 0.63 \\
\hline 58 & Sp & - & 2.28 & 0.56 \\
\hline 59 & Sp & - & 2.28 & 0.63 \\
\hline 61 & Sp & - & 2.28 & 0.54 \\
\hline
\end{tabular}

where $\left\langle>-\right.$ averaging operator, $\eta_{H}$ - Hilbert transform of the free surface elevations, $\sigma$ - standart deviation of free surface elevations. 


\begin{tabular}{|l|l|l|l|}
\hline \multicolumn{4}{|l|}{ Table 4. Parameters of breaking waves in laboratory experiment } \\
\hline $\begin{array}{l}\text { No. of } \begin{array}{l}\text { Type of } \\
\text { weasurement } \\
\text { series }\end{array} \\
\text { breaking }\end{array}$ & $\begin{array}{l}\text { Period, } \\
T, \mathrm{~s}\end{array}$ & $\mathrm{~V}=H_{s} / h_{b}$ \\
\hline 42 & $\mathrm{Pl}$ & 6.0 & 0.97 \\
\hline 41 & $\mathrm{Pl}$ & 7.0 & 1.02 \\
\hline 41 & $\mathrm{Pl}-\mathrm{Sp}$ & 7.0 & 0.57 \\
\hline 40 & $\mathrm{Pl}$ & 6.5 & 1.05 \\
\hline 31 & $\mathrm{Pl}$ & 5.0 & 0.70 \\
\hline 32 & $\mathrm{Pl}$ & 5.0 & 1.00 \\
\hline 34 & $\mathrm{Pl}$ & 7.0 & 1.10 \\
\hline 36 & $\mathrm{Pl}$ & 7.0 & 0.85 \\
\hline 37 & $\mathrm{Pl}$ & 7.0 & 0.92 \\
\hline 39 & $\mathrm{Pl}$ & 7.0 & 1.03 \\
\hline 65 & $\mathrm{Pl}$ & 7.0 & 0.96 \\
\hline 64 & $\mathrm{Pl}$ & 6.5 & 0.96 \\
\hline 63 & $\mathrm{Pl}$ & 7.0 & 0.91 \\
\hline 62 & $\mathrm{Pl}$ & 7.0 & 0.93 \\
\hline 60 & $\mathrm{Pl}$ & 6.0 & 0.99 \\
\hline 58 & $\mathrm{Pl}$ & 6.0 & 1.06 \\
\hline 58 & $\mathrm{Sp}$ & 6.0 & 0.52 \\
\hline 57 & $\mathrm{Sp}-\mathrm{Pl}$ & 6.0 & 0.88 \\
\hline 57 & $\mathrm{Sp}$ & 6.0 & 0.64 \\
\hline 32 & $\mathrm{Sp}$ & 5.0 & 0.66 \\
\hline 40 & $\mathrm{Sp}$ & 6.5 & 0.45 \\
\hline 36 & $\mathrm{Sp}$ & 7.0 & 0.54 \\
\hline 37 & $\mathrm{Sp}$ & 7.0 & 0.52 \\
\hline 39 & $\mathrm{Sp}$ & 7.0 & 0.43 \\
\hline 65 & $\mathrm{Sp}$ & 7.0 & 0.55 \\
\hline 64 & $\mathrm{Sp}$ & 6.5 & 0.65 \\
\hline 62 & $\mathrm{Sp}$ & 7.0 & 0.55 \\
\hline 60 & $\mathrm{Sp}$ & 6.0 & 0.49 \\
\hline & & & \\
\hline & & & \\
\hline & & & \\
\hline
\end{tabular}

On Fig. 9 the dependence of asymmetry coefficient on biphase for breaking waves are shown. Parameters of experimental breaking waves are in Tables 3 and 4. It is well visible that a) asymmetry of breaking waves linear depends on biphase and b) biphase of breaking waves changes from $[-\pi / 2, \pi / 6]$ and its value depends on type of wave breaking. The biphase of most of spilling breaking waves in changes in $[-\pi / 3, \pi / 6]$ and only the biphase of most of plunging breaking waves tends to $-\pi / 2$ and changes in $[-\pi / 2,-\pi / 6]$.

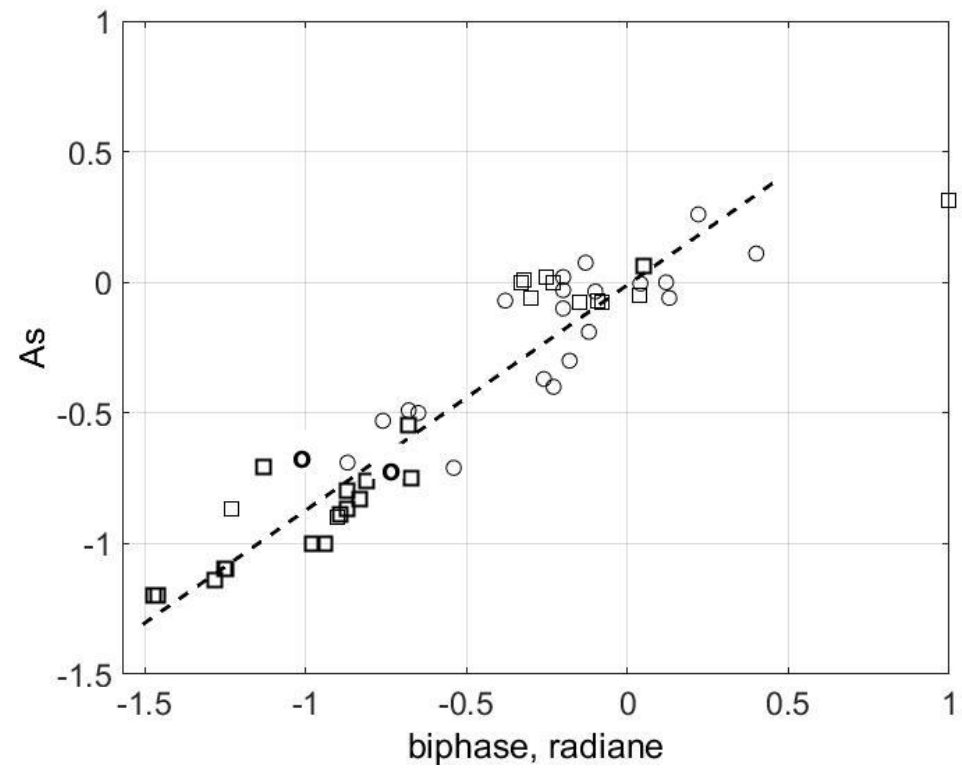

Figure 9. Asymmetry of breaking waves on the biphase. Circles - data of laboratory experiment, squares field data. Bold symbols - plunging breaking waves, thin symbols - spilling breaking waves. 
As it was demonstrated in (Saprykina, et al., 2016; Saprykina et al, 2017) breaking index $\gamma-$ relation between wave height and depth of wave breaking - depends on type of wave breaking and asymmetry of waves. Therefore the biphase can be usefull to define breaking index and breaking type.

On Fig. 10 the dependence of breaking index on biphase are shown. The breaking index increases with decreasing of biphase in general. But two main tendencies can be observed. For waves breaking mainly by spilling type (biphase more then $-\pi / 3$ ) the breaking index changes slightly, and for example, its value can be accepted to the constant which isn't depending on the biphase. And for waves breaking mainly by plunging type (biphase less then $-\pi / 3$ ) the breaking index increases with decreasing of the biphase. So, if the biphase is known there is possible to define type of breaking and breaking index.

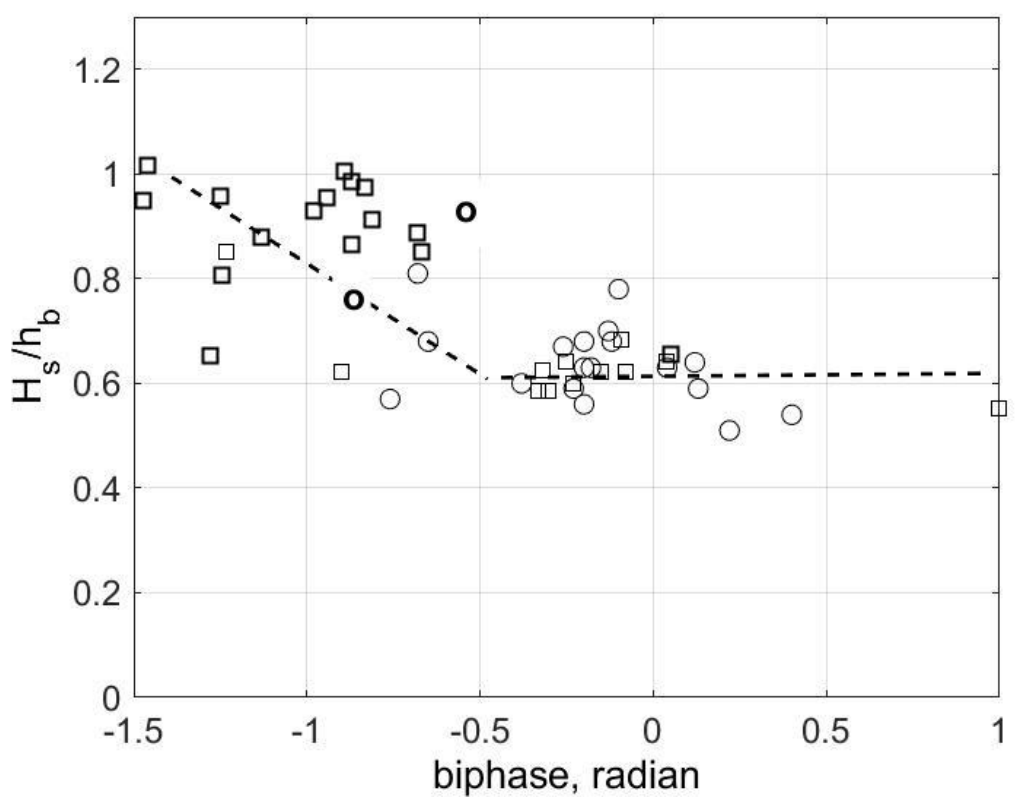

Figure 10. The breaking index on the biphase. Circles - data of laboratory experiment, squares - field data. Bold symbols - plunging breaking waves, thin symbols - spilling breaking waves.

\section{CONCLUSIONS}

1. In nonlinear dispersive waves, a change in the biphase strictly follows changes in the amplitudes of the first and second harmonics and the periodicity of energy exchange between them. Here, the biphase varies in the interval $[-\pi / 2, \pi / 2]$.

2. Eq.4, widely applied in practice, of the dependence of the biphase on the Ursell number is unsuitable for correctly calculating the biphase during wave propagation over an sloping bottom in the coastal zone, because it does not take into account periodic energy exchange between the nonlinear harmonics during near resonant triad interactions. As another approach, a simple linear approximation of the biphase from relative wave run $\Delta l$ (the ratio of the current distance to the coast to the possible length of spatial period of the energy exchange) is suggested. For $\Delta l<1$, the biphase is approximately equal to $\pi / 2 * \Delta l-\pi / 2$, and for $\Delta l>5$, the biphase is equal to zero. For $1<\Delta l<5$, the biphase can be unambiguously parameterized.

3. From the available experimental data, a dependence of biphase measurements on the wave transformation scenario and the possibility of constructing the parameterization of the biphase from $\Delta l$ for each of the scenarios are observed. However, the observed regularities require additional experimental verification.

4. The biphase of breaking waves defines breaking index and breaking type.

Our research and the obtained parameterizations of the biphase can be used for modeling the sea state in the coastal zone and in problems on predicting the development of coasts under the impact of storm waves. 


\section{ACKNOWLEDGMENTS}

The study was supported by the Russian Science Foundation (project no. 14-50-00095); M.N. Shtremel's participation was supported by the Russian Foundation for Basic Research (project no. 1405-00696).

\section{REFERENCES}

Antsyferov S. M., R. D. Kosyan, S. Yu. Kuznetsov, and Ya. V. Saprykina. 2005. Physical grounds for the formation of the sediment flux in the coastal zone of a nontidal sea, Oceanology 45 (1), S183S190.

Bagnold R. A. 1966. An approach to the sediment transport problem from general physics, US Geological Survey, Professional Paper, No. 422_I.

Bailard J. A. and D. L. Inman. 1981. An energetic bedload model for a plane sloping beach: Local transport, J. Geophys. Res. 86, 2035-2043.

Doering J. C. and A. J. Bowen. 1986. Shoaling surface gravity waves: a bispectral analysis, Proceedings of 20th Conf. on Coastal Engineering, Taipei, 150-162.

Doering J. C. and A. J. Bowen. 1995. Parametrization of orbital velocity asymmetries of shoaling and breaking waves using bispectral analysis, Coastal Eng., 26, 15-33.

Eldeberky Y. and J. Battjes. 1995. Parametrization of triad interactions in wave energy models, Proceedings of the International Conf. on Coastal Research in Terms of Large Scale Experiments “Coastal Dynamics'95, 140-148.

Elgar S. and R. T. Guza. 1985. Observation of bispectra of shoaling surface gravity waves, J. Fluid Mech. 161, 425-448.

Elgar S. and R. T. Guza.1985. Shoaling gravity waves: comparison between field observations, linear theory and a nonlinear model, J. Fluid Mech. 158, 47-70.

Elgar S. and R. T. Guza. 1986. Nonlinear model predictions of bispectra of shoaling surface gravity waves, J. Fluid Mech. 167, 1-18.

Hasselmann K., W. Munk, and G. MacDonald. 1963. Bispectra of ocean waves, Proceedings of the Symp. on Time Series Analysis, 125-139.

Kim Y. and E. Powers. 1979. Digital bispectral analysis and its application to non-linear wave interaction, IEEE Trans. Plasma Sci. 1, 120-131.

Kuznetsov S. and Y. Saprykina. 2012. Secondary waves in coastal zone: physical mechanisms of formation and possible application for coastal protection. Proceeding of $33^{\text {th }}$ International Conference on Coastal Engineering, dx.doi.org/10.9753/icce.v33.waves.12

Kuznetsov S.Yu., Ya.V. Saprykina, B.V. Divinskii, M.N. Shtremel, 2015. Spectral structure of breaking waves. Proceedings IMAM 2015, Towards Green Marine Technology and Transport, Taylor \& Francis Group, London, 853-858.

Madsen P. A. and O. R. Sorensen. 1993. Bound waves and triad interactions in shallow water, J. Ocean Eng. 20 (4), 359-388.

Saprykina Ya. V., S. Yu. Kuznetsov, Zh. Cherneva, and N. Andreeva. 2009. Spatio-temporal variability of the amplitude-phase structure of storm waves in the coastal zone of the sea, Oceanology, 49 (2), 182-192.

Saprykina Ya. V., S. Yu. Kuznetsov, N. K. Andreeva, and M. N. Shtremel. 2013. Scenarios of nonlinear wave transformation in the coastal zone, Oceanology, 53 (4), 422-431.

Saprykina Ya.V., S.Yu.Kuznetsov, B.V.Divinskii. 2017. The influence of the processes of nonlinear transformations of waves in the coastal zone on the height of breaking waves, Oceanology, 57, in press

Saprykina Y., B. Divinskiy, S. Kuznetsov. 2016. Relation of wave breaking criteria with spectral structures of waves. Proceedings of the 6th International Conference on the Application of Physical Modelling in Coastal and Port Engineering and Science (Coastlab16), Ottawa, Canada, http://rdio.rdc.uottawa.ca/publications/coastlab16/coastlab79.pdf

SWAN, Technical documentation, 2006. http://www.swan.tudelft.nl 